\title{
Fibrosis and Damage Markers in Occupational Interstitial Lung Diseases
}

Authors:

Disclosure:

Recieved:

Accepted:

Keywords:

Citation:
*Galina P. Orlova, ${ }^{1}$ Elena A. Surkova, ${ }^{2}$ Sergey V. Lapin ${ }^{2}$

1. Research Institute for Interstitial and Orphan Lung Diseases, Pavlov First SaintPetersburg State Medical University, Saint-Petersburg, Russia

2. Laboratory for the Diagnosis of Autoimmune Diseases of the Scientific and Methodological Center for Molecular Medicine, Pavlov First Saint-Petersburg State Medical University, Saint-Petersburg, Russia

${ }^{*}$ Correspondence to galorlova@mail.ru

The authors have declared no conflicts of interest.

14.05.19

13.08 .19

Biomarkers, disease activity, occupational alveolitis, pneumoconiosis.

EMJ Respir. 2019;DOI/10.33590/emjresp/19-00103.

\section{Abstract}

The investigation of biomarkers for fibrosis and lung tissue damage is very important for the diagnosis and prognosis of interstitial lung disease, as well as treatment strategies. The biomarkers secreted by Type II pneumocytes and cells of the bronchiolar epithelium are most reflective of the formation of pulmonary fibrosis and the degree of damage to the lung tissue. The levels of Krebs von den Lungen-6 (KL-6), alveolomucin (mucin-antigen 3EG5), and Clara cell secretory protein in occupational interstitial lung diseases (e.g., exogenous alveolitis, pneumoconiosis), caused by exposure to organic and inorganic factors, were analysed in dependence of disease activity phase. The level of alveolomucin in pneumoconiosis and in occupational alveolitis remission may reflect the extent of pulmonary fibrosis, which is a prognostic sign of the outcome of the disease. Higher specificity and lower sensitivity of alveolomucin compared to KL-6 can be used as a screening test for exogenous alveolitis. KL- 6 and alveolomucin are more useful biomarkers than Clara cell secretory protein for diagnosis, exacerbation, and progression of occupational alveolitis.

\section{INTRODUCTION}

Interstitial lung disease (ILD) is a group of heterogeneous disorders with morbid conditions characterised by inflammation, including alveolitis and granulomatosis that lead to the subsequent development of pulmonary fibrosis. ${ }^{1}$ Interstitial pneumonias, an ILD group member, vary in their aetiology and pathogenesis and lead to predominant damage in the pulmonary interstitium and the development of progressive pulmonary fibrosis, resulting in honeycombing. Interstitial pneumonias include idiopathic

interstitial pneumonia, exogenous allergic and toxic alveolitis, and syndromes of ILD associated with other diseases. Pneumoconioses (PC) are an ILD with characteristic granuloma formation in response to occupational factors. ${ }^{1}$

Inhaled organic or inorganic substances, such as allergens, toxins, fibrogenic dust, and certain drugs, can affect the interstitium of the lung, alveolar epithelium, and pulmonary capillary endothelium. The increased expression of certain proteins by alveolar cells and bronchiolar epithelial cells following cellular injury may be used as a marker for ILD. 
Serum biomarkers, high resolution CT (HRCT), and lung function tests may be used for diagnostic purposes, to give information about inflammation activity and responsiveness to therapy, and risk of ILD progression. ${ }^{2}$ Biomarkers for Type II pneumocytes are of particular interest because these cells are prevalent in the pathophysiological development of ILD, scilicet injury, and remodelling of lung tissue. ${ }^{3,4}$ The most widely used biomarkers derived from Type II pneumocytes are KL-6 and the surfactant proteins SP-A and SP-D. Decreased alveolomucin (mucin-antigen 3EG5) glycoprotein expression on the apical surface of Type II pneumocytes has also been used as an ILD disease biomarker.

\section{AIM}

To reveal the role of the fibrosis biomarkers alveolomucin and KL-6, and the destructive respiratory tract epithelial biomarker Clara cell secretory protein (CC16) in the progression of ILD.

\section{METHODS}

A total of 13 PC, 26 exogenous toxic alveolitis (ETA), and 20 exogenous allergic alveolitis (EAA) patients (mean age $54.8 \pm 0.7$ years, 59.4 \pm 2.5 years, and 57.1 \pm 2.1 years, respectively) were investigated. Nine patients in the PC group were exposed to silica and the remaining four were exposed to welding aerosols; in the ETA group, four patients were exposed to metals, four to irritants, ten to drugs, and eight to dyes and solvents; and in the EAA group, five participants had exposure to animals such as birds, eight to dyes and solvents, and the remaining patients had exposure to antibiotics. Ten (38\%) patients with ETA, three (15\%) with EAA, and seven (54\%) with PC were smokers. All procedures were performed in accordance with national guidelines for the provision of medical care to humans. Blood samples were collected from all subjects. Levels of CC16, alveolomucin, and KL-6 in the serum were detected using ELISA, and further quantified using the test systems BioVendor (Brno, Czech Republic) for CC16 and KL-6, and XEMA (Moscow, Russia) for alveolomucin.

The normal range for $\mathrm{CC} 16$ in the serum is 3.7-23.2 $\mathrm{ng} / \mathrm{mL},<70 \mathrm{U} / \mathrm{mL}$ for alveolomucin, and $<500 \mathrm{U} /$ $\mathrm{mL}$ for KL6. Statistical analysis was performed using GraphPad Prism 6.0 Software (San Diego, California, USA). Comparisons between groups were made using the nonparametric MannWhitney $U$ test. A two-sided $p$-value $<0.05$ indicated statistical significance. Data were expressed as mean \pm standard deviation $(M \pm S D)$. The activity of the disease and its progression was evaluated by the clinical manifestations, HRCT patterns, and lung function tests.

The serum level of an ideal marker should increase in the presence of the disease (sensitivity), and not increase in the absence of the disease (specificity). Sensitivity and specificity calculated at various cut-off points give rise to a receiver operating-characteristic (ROC) curve. As a result, the most useful biomarkers will have the largest area under the curve (AUC).

\section{RESULTS}

Of 26 patients with ETA and 20 patients with EAA, the disease was active in 14 ETA patients and 14 EAA patients; remission of the disease was observed in 12 patients with ETA and in six patients with EAA. The active stage of the disease was characterised by progressive dyspnoea; fever, as seen in four ETA patients; weight loss, as seen in three ETA patients; increased erythrocyte sedimentation rate in the blood; the appearance or increase of alveolar nodules (infiltration, ground-glass opacities); and interstitial changes, such as reticulation, traction bronchiectasis, and honeycombing.

Activation of the disease leads to fibrosis progression and decreased diffusing capacity of the lungs (Table 1). Alveolitis remission was deemed through clinical, functional, and HRCT stabilisation, or through improvement with glucocorticosteroids and plasmapheresis therapy. The severity of the disease was determined by the degree of damage to organs and/or systems of the human body, or a violation of their functions due to the disease. Severity was due to the degree of pathological activity or the response to therapy, i.e., the degree of progression of the fibrosing process in the lungs. Serum KL6 in patients with active ETA and EAA was significantly higher than those in remission (Table 1). In patients with active ETA, the level of KL- 6 was not significantly higher than in patients with EAA $(p<0.05)$. 
Table 1: Alveolomucin, KL-6, CC16 serum levels, high resolution CT patterns and diffusing capacity of the lungs at patients with occupational alveolites.

\begin{tabular}{|c|c|c|c|c|c|c|}
\hline & \multicolumn{3}{|c|}{ EAA } & \multicolumn{3}{|c|}{ ETA } \\
\hline & $\begin{array}{l}\text { Activity } \\
(n=14)\end{array}$ & $\begin{array}{l}\text { Remission } \\
\quad(n=6)\end{array}$ & $\mathrm{p}$ & $\begin{array}{l}\text { Activity } \\
(n=14)\end{array}$ & $\begin{array}{l}\text { Remission } \\
\quad(n=12)\end{array}$ & $\mathrm{p}$ \\
\hline Men & 1 & 0 & - & 6 & 8 & - \\
\hline Women & 13 & 6 & - & 8 & 4 & - \\
\hline Age (years) & $59.29 \pm 2.23$ & $52.00 \pm 3.70$ & - & $62.47 \pm 4.05$ & $57.50 \pm 2.66$ & - \\
\hline ESR $(\mathrm{mm} \mathrm{Hg})$ & $18.29 \pm 3.23$ & $12.33 \pm 3.16$ & - & $28.00 \pm 4.74$ & $10.83 \pm 1.71$ & $p<0.02$ \\
\hline $\begin{array}{l}\text { HRCT infiltration } \\
n(\%)\end{array}$ & $1(7 \%)$ & - & - & $3(21 \%)$ & - & - \\
\hline $\begin{array}{l}\text { HRCT - GGO } \\
\text { n (\%) }\end{array}$ & $7(50 \%)$ & $2(33 \%)$ & - & $8(57 \%)$ & $2(17 \%)$ & $p<0.02$ \\
\hline $\begin{array}{l}\text { HRCT foci } \\
n(\%)\end{array}$ & $3(21 \%)$ & $2(33 \%)$ & - & $4(29 \%)$ & - & - \\
\hline $\begin{array}{l}\text { HRCT reticulation } \\
n(\%)\end{array}$ & $13(93 \%)$ & $4(67 \%)$ & $\mathrm{p}<0.2$ & $12(86 \%)$ & $11(92 \%)$ & - \\
\hline $\begin{array}{l}\text { HRCT traction } \\
\text { bronchiectasis } \\
\mathrm{n}(\%)\end{array}$ & $3(21 \%)$ & - & - & $3(21 \%)$ & - & - \\
\hline $\begin{array}{l}\text { HRCT } \\
\text { honeycombing } \\
\text { n (\%) }\end{array}$ & $5(35 \%)$ & $1(17 \%)$ & - & $3(21 \%)$ & - & - \\
\hline DLco \% Predicted & $48.96 \pm 4.28$ & $58.12 \pm 4.66$ & $p<0.2$ & $50.29 \pm 4.67$ & $63.58 \pm 4.58$ & $p<0.1$ \\
\hline $\begin{array}{l}\text { Alveolomucin (U/ } \\
\mathrm{mL} \text { ) }\end{array}$ & $82.17 \pm 10.19$ & $19.22 \pm 5.40$ & $p<0.001$ & $90.40 \pm 8.86$ & $39.03 \pm 5.01^{* *}$ & $p<0.001$ \\
\hline $\mathrm{KL}-6(\mathrm{U} / \mathrm{mL})$ & $1900.10 \pm 381.10$ & $498.50 \pm 230.50$ & $p<0.01$ & $2594.20 \pm 677.10$ & $460.50 \pm 95.70$ & $p<0.005$ \\
\hline CC16 (ng/mL) & $22.11 \pm 5.65$ & $9.40 \pm 3.30$ & $p<0.1$ & $21.58 \pm 4.20$ & $10.66 \pm 2.68$ & $p<0.005$ \\
\hline
\end{tabular}

${ }^{* *} \mathrm{p}<0.02$, when compared with EAA remission

DLco: diffusing capacity of the lungs; EAA: exogenous allergic alveolitis; ETA: exogenous toxic alveolitis; GGO: ground-glass opacity; HRCT: high resolution CT.

Alveolomucin levels in the blood of patients with ETA and EAA was higher in the active period than in remission $(p<0.001)$ (Table 1$)$. In remission, the level of alveolomucin in ETA patients was higher $(p<0.02)$ than in patients with EAA. These data suggest the instability of ETA remission, which may be caused by minimal and maintained activity of the inflammatory processes. The levels of Clara cells and CC16 protein reflect epithelial damage to the lung caused by occupational alveolite activity. Levels of CC16 were higher in patients with the active disease than for those in remission for both ETA and EAA (Table 1). In patients with ETA, CC16 levels were significantly higher in those with the active disease $(21.58 \pm 4.2$ $\mathrm{ng} / \mathrm{mL}$ ) than those in remission $(10.66 \pm 2.68 \mathrm{ng} /$ $\mathrm{mL})(\mathrm{p}<0.005)$. The increased level of serum CC16 between patients with active EAA and EAA in remission was not statistically significant $(p<0.1)$. This may be due to the small number of patients with EAA in remission $(n=6)$. However, the high level of $\mathrm{CC} 16$ at remission was found more frequently (0.17) in EAA patients versus ETA patients (0.08). According to the literature, when exposed to toxic factors, low levels of CC16 in the serum may be caused by a decrease in the 
number of Clara cells, changes in the permeability of the alveolar epithelium, and potentially by reduced expression of CC16 in airway epithelium. 5,6

In the PC group, the serum concentration of alveolomucin $(40.89 \pm 3.49 \mathrm{U} / \mathrm{mL}, \mathrm{p}<0.001$ versus ETA, EAA), KL-6 (429.60 $\pm 145.10 \mathrm{U} / \mathrm{mL}, \mathrm{p}<0.002$ versus EAA, $\mathrm{p}<0.005$ versus $\mathrm{ETA}$ ) and $\mathrm{CC} 16$ ( $7.541 \pm 0.87 \mathrm{ng} / \mathrm{mL}, \mathrm{p}<0.02$ versus EAA, $\mathrm{p}<0.005$ versus ETA) was significantly lower than active occupational alveolitis. In remission, KL-6 and CC16 levels were not different from PC. The level of alveolomucin was not significantly different in participants with PC compared to ETA in remission, but was significantly higher than the group with EAA in remission $(p<0.005)$. This confirms the theory that alveolomucin is a marker not only for alveolitis activity in which signs of HRCT fibrosis are detected much more often (Table 1), but also of pneumofibrosis in the whole, as in PC. Thus, with adequate treatment of the acute and subacute forms of EAA, pronounced, dynamic changes in the lungs are possible, potentially resulting in pulmonary pattern normalisation. Pneumoconiosis on the other hand is characterised by widespread diffuse or focal pulmonary fibrosis. In ETA, the pathological process in the lungs proceeds more aggressively than in EAA and pulmonary fibrosis can develop even during glucocorticosteroid therapy. Further research is required to support this theory.

Sensitivity and specificity for KL-6 and alveolomucin as biomarkers were calculated for the active disease phase in exogenous alveolitis (the clinical and radiological changes were used as a control test), producing the following results. Sensitivity for KL-6 was $85 \%$ and specificity was calculated as $67 \%$ in EAA. Sensitivity and specificity for alveolomucin in EAA was calculated as $54 \%$ and $100 \%$, respectively. The sensitivity and specificity of KL-6 as a biomarker for ETA were $93 \%$ and 50\%, respectively, and for alveolomucin values were calculated as $67 \%$ and $92 \%$, respectively. Alveolomucin, like $\mathrm{KL}-6$, is a biomarker for fibrosis in EAA and ETA and had a higher specificity compared with KL-6. Therefore, determination of levels of alveolomucin in blood could allow diagnosis screening for exogenous alveolitis. Measurement of KL- 6 levels in the blood could determine diagnosis and progression of exogenous alveolitis (Figure 1).

There were no significant differences in ROC AUC between alveolomucin and KL-6, which were 0.92 and 0.90 , respectively. Both were higher than ROC AUC for CC16, measured at 0.78 .

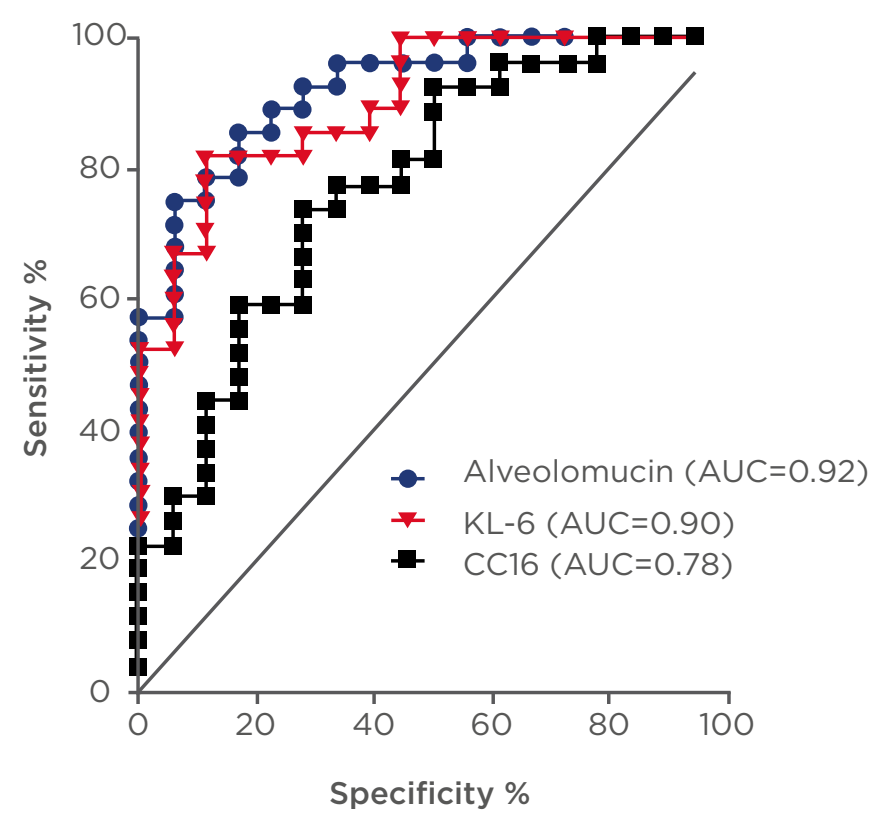

Figure 1: Receiver operating characteristic analysis for alveolomucin, KL-6, and CC16 in patients with occupational alveolitis.

AUC: area under the curve; CC16: Clara cell secretory protein; KL-6: Krebs von den Lungen-6. 


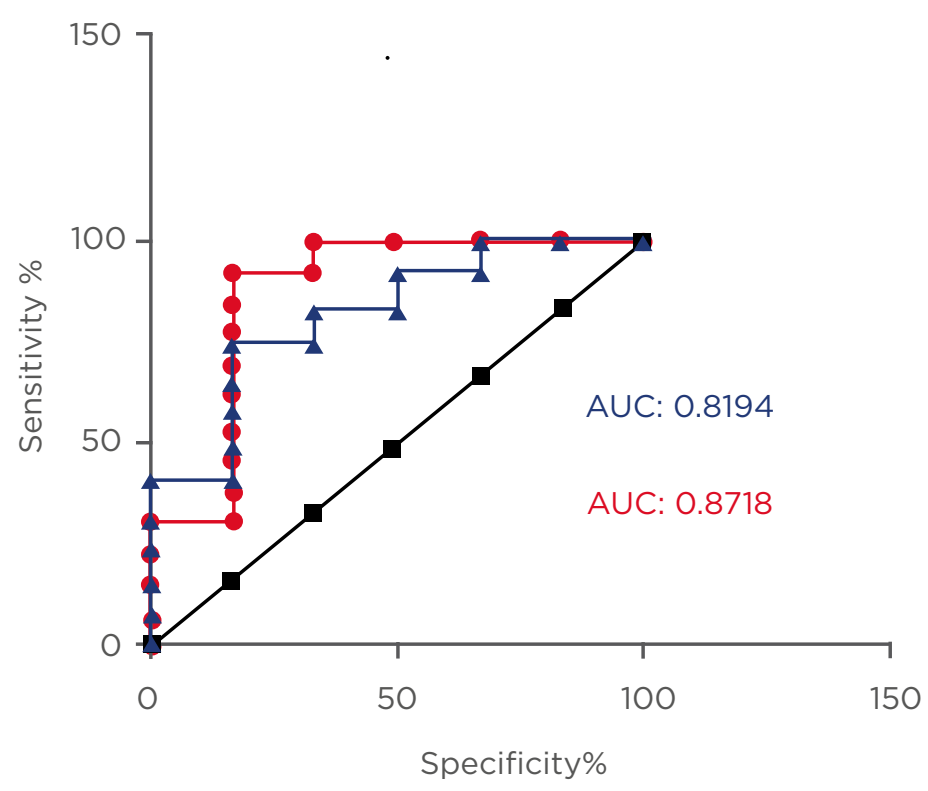

Figure 2: Receiver operating characteristic

analysis for alveolomucin at pneumoconiosis and remission of alveolitis.

AUC: area under the curve.

These data suggest that all the biomarkers tested may be useful in valuation of exogenous alveolitis activity. For occupational alveolitis, KL- 6 had a specificity of $94.0 \%$ and sensitivity of $66.7 \%$, alveolomucin showed $94.4 \%$ specificity and $75.0 \%$ sensitivity, and CC16 had a specificity of $94.0 \%$ and sensitivity of $29.6 \%$. Because of the low sensitivity of CC16, the authors concluded that alveolomucin and KL-6 are more preferable than $\mathrm{CC} 16$ as markers for exogenous alveolitis progression.

According to ROC analysis, AUC for alveolomucin in $P C$ and ETA in remission was higher than in EAA remission (Figure 2). The results show that in ETA remission, low levels of fibrosis may occur, so as the HRCT reticulation were revealed in 92\% of patients (Table 1) and alveolomucin can be used as a biomarker for fibrosis or as a predictor of instability in the disease. Determination of alveolomucin levels in the blood in patients with ETA is useful for diagnosing minimal activity of ETA even in remission.

\section{DISCUSSION}

\section{KL-6 in Fibrotic Lung Diseases}

$\mathrm{KL}-6$ is a high-molecular-weight (200 kDa) glycoprotein and is classified as human mucin. It is expressed on the apical surface cellular membrane. Type II pneumonocytes are activated after the death of Type I pneumonocytes during the first stage of lung tissue injury. In ILD, Type II pneumocytes are damaged and inflammatory mediators such as proteases, TGF- $\beta 1$, proinflammatory cytokines, and TNF-a affect the integrity of the alveolar epithelium leading to a marked increase in paracellular permeability to protein and causes significant alveolar epithelial damage. Therefore, increased levels of KL-6 in serum reflect the destruction of the parenchyma and lung structure, and the increased permeability of the blood-air barrier. Activation of KL-6 allows for migration of lung and skin fibroblasts, suggesting its role in lung fibrosis. $^{7} \mathrm{KL}-6$ demonstrates chemotactic and antiapoptotic effects on fibroblasts, and expression of activated collagen Types I and III. Thus, KL-6 may be one of the key molecules involved in intra-alveolar pulmonary fibrosis. ${ }^{8}$ 
There have been several published papers reporting estimated values for $\mathrm{KL}-6$ as a diagnostic and prognostic marker for ILD disease progression. High levels of KL-6 are seen in the sera of patients with idiopathic interstitial pneumonia, drug-induced ILD, pulmonary sarcoidosis, and connective tissue diseaseassociated ILD. ${ }^{9,10}$ It has been reported that KL-6 has a high level of sensitivity and specificity for ILD such as idiopathic pulmonary fibrosis (IPF), and serum levels of KL-6 are $70-100 \%$ significantly higher in IPF. Analysis of the relationship between KL- 6 serum levels and disease activity suggests that KL-6 serum levels are significantly correlated with the severity of the interstitial pneumonia and IPF.11-13

KL-6 serum levels are elevated in patients with season-type EAA. Several reports demonstrate that KL-6 serum levels were significantly higher in patients with farmer's lung disease compared to healthy farmers. ${ }^{14,15} \mathrm{KL}-6$ was used as a predictor for the occurrence, progression, and activity of drug-induced interstitial lung disease. ${ }^{16,17}$ The data from the authors' report suggest the high sensitivity and specificity for KL-6 as a biomarker for activity of exogenous alveolitis (ETA and EAA). Serum levels of KL- 6 reflect the activity of fibrotic pulmonary processes. Elevated serum $\mathrm{KL}-6$ in IPF patients $>500 \mathrm{U} / \mathrm{mL}$ are associated with progression of the disease and increased mortality. ${ }^{18,19}$ Integration of KL-6 as a marker of ILD into clinical practice however remains limited.

\section{Alveolomucin in Fibrotic Lung Diseases}

Alveolomucin is also expressed at the Type II pneumocytes apical surface cellular membrane. Increased levels of serum alveolomucin could reflect the degree of proliferation of Type II alveolocytes in lung fibrosis. Nevertheless, alveolomucin serum concentration is rarely used to detect disease activity and progression. Alveolomucin plasma levels are elevated in patients with interstitial pneumonitis and strongly correlates with the degree of disease activity. ${ }^{20}$ At the same time, the data concerning these biomarkers, such as their prognostic value pertaining to severity and activity of occupational ILD, is not enough.

Research detailing alveolomucin concentrations in patients with occupational alveolitis is limited. In this study, the authors demonstrated higher levels of alveolomucin in the blood serum of patients with ETA and EAA during exacerbation of the disease. Analysis of ROC curves and cutoff values demonstrate that there is no distinct differentiation between alveolomucin and KL-6. Both alveolomucin and KL-6 can be used as serum biomarkers for occupational alveolitis activity. Higher levels of alveolomucin in PC and ETA in remission compared to EAA in remission may be explained by the remaining minimal active fibrogenic process in PC and ETA (even with clinical and radiological ETA remission) and the extent of pulmonary fibrosis in these diseases compared to EAA in remission. Further research is required in support of this.

\section{CC16 in Fibrotic Lung Diseases}

Clara cells are the major cell type of bronchiolar epithelium and help to maintain lung homeostasis. Clara cells in bronchioles are self-renewing and contribute to the long-term maintenance and repair of Type II alveolocytes in lung epithelium after severe pulmonary damage. CC16 is a lowmolecular-weight protein of $16 \mathrm{kDa}$ secreted into the lumen of the respiratory tract. CC16 serves as an immunosuppressive, anti-inflammatory mediator in the lung. High levels of CC16 in the serum follow impairment of the air-blood barrier and pulmonary fibrosis. It is a good marker of destructive processes in the respiratory tract epithelium and high levels in the blood indicate lung injury in ILD. 5,21,22

Regeneration of alveolar epithelia following severe toxic or allergic pulmonary damage is critical for lung function. Clara cells are capable of self-renewal for the repair of bronchioles and contribute to respiratory epithelium repair. ${ }^{21}$ The authors suggest that CC16 levels in the blood may be associated with the degree of repair processes in the lungs and, thus, may serve as a useful biomarker for ETA and EAA. Higher CC16 concentrations were found in the blood of patients with ETA and EAA during exacerbation of disease compared to the disease in remission. There were no differences between levels of CC16 in the serum of patients with ETA and EAA. ROC curve analysis revealed AUC data was lower for CC16 than for alveolomucin and KL-6. In conditions of occupational exposure, toxic and allergic dusts cause different changes in the respiratory system and in serum biomarkers, namely CC16, and may alter the function of Clara cells. The relationship 
between dust exposure and disease is dependent on the intervention mechanism of the agent. Previous studies have demonstrated that distal airspace epithelial cells were altered by asbestosis and CC16 level was increased in asbestos-exposed workers. ${ }^{23}$ Furthermore, CC16 synthesis in smokers with asbestosis was significantly higher versus healthy smokers but lower than in non-smoker patients with asbestosis. According these results, CC16 may be employed as a peripheral marker of the lung epithelium function in smokers and nonsmoker with occupation diseases..$^{23,24}$

At the same time, there was an inverse relationship between $\mathrm{CC} 16$ and $\mathrm{Al}_{2} \mathrm{O}_{3}$ concentrations in blood serum of aluminium production workers and proved to damage Clara cells in response to toxic substances.5,21,22,25 Chronic allergyinduced metaplasia affects CC16 as a defensive mechanism. Effects of chronic allergy significantly reduced CC16 in bronchoalveolar lavage; however, anti-inflammatory treatments were able to reestablish the normal phenotype and function of Clara cells. ${ }^{6}$

\section{CONCLUSION}

Alveolomucin and KL-6 can be used as a serum biomarker of occupational alveolitis activity. KL-6 and alveolomucin were more useful biomarkers in the diagnosis and progression of exogenous alveolitis when compared with CC16. The level of alveolomucin in the period of remission in alveolitis and in PC may reflect the extent of pulmonary fibrosis, which is a prognostic sign of the outcome of the disease. The higher specificity and lower sensitivity of alveolomucin compared with KL-6 may allow it to be used as an exogenous alveolitis screening test. It is advisable to monitor the level of alveolomucin and KL-6 in the blood, which would make it possible to promptly diagnose and optimise therapy for ETA and EAA.

\section{References}

1. II'kovich MM, "Interstitial and orphan lung diseases. Specialist Library," II'kovich MM (eds.), [Interstitial and orphan lung diseases] (2019), Moscow: GEOTAR-Media, pp.14-27. (In Russian).

2. Kinnula VL et al. Proteomic approaches for studying human parenchymal lung diseases. Expert Rev Proteomics. 2009;6(6):619-29.

3. Nukiwa T. "The role of biomarkers in management of interstitial lung disease: implication of biomarkers derived from Type II pneumocytes," Du Bois RM, Richeldi L (eds.), Interstitial lung disease (2009), UK: ERS Journals Ltd, pp.47-66.

4. Hu $Y$ et al. Serum krebs von den lungen- 6 level as a diagnostic biomarker for interstitial lung disease in Chinese patients. Clin Respir J. 2017;11(3):337-45.

5. Kropski J et al. Clara cell protein (CC16), a marker of lung epithelial injury, is decreased in plasma and pulmonary edema fluid from patients with acute lung injury. Chest. 2009;135(6):1440-7.

6. Roth FD et al. Restoration of the normal clara cell phenotype after chronic allergic inflammation. Int J Exp Pathol. 2013;94(6):399-411.

7. Hesselstrand $\mathrm{R}$ et al. Biomarkers from bronchoalveolar lavage fluid in systemic sclerosis patients with interstitial lung disease relate to severity of lung fibrosis. Respir Med. 2013;107(7):1079-86.

8. $\mathrm{Xu} L$ et al. $\mathrm{KL}-6$ regulated the expression of HGF, collagen and myofibroblast differentiation. Eur Rev Med Pharmacol Sci. 2013;17(22):30737.

9. Ishikawa $\mathrm{N}$ et al. Utility of KL-6/ MUC1 in the clinical management of interstitial lung diseases. Respir Investig. 2012;50(1):3-13.

10. Lee JS et al. Serum KL- 6 levels reflect the severity of interstitial lung disease associated with connective tissue disease. Arthritis Res Ther. 2019;21(1):58.

11. Ichiyasu $\mathrm{H}$ et al. Pneumocyte biomarkers KL- 6 and surfactant protein $\mathrm{D}$ reflect the distinct findings of high-resolution computed tomography in non-specific interstitial pneumonia. Respiration. 2012;83(3):190-7.

12. Ley $\mathrm{B}$ et al. Molecular biomarkers in idiopathic pulmonary fibrosis. Am J Physiol Lung Cell Mol Physiol. 2014;307(9):L681-91.

13. Hanaoka $M$ et al. KL-6 is a longterm disease-activity biomarker for interstitial lung disease associated with polymyositis/dermatomyositis, but is not a short-term diseaseactivity biomarker. Mod Rheumatol. 2019;29(4):625-32.

14. Takahashi T et al. Serum KL-6 concentrations in dairy farmers.
Chest. 2000;118(2):445-50.

15. Ohnishi $\mathrm{H}$ et al. Seasonal variation of serum KL-6 concentrations is greater in patients with hypersensitivity pneumonitis. BMC Pulm Med. 2014;7(14):129.

16. Kawase $\mathrm{S}$ et al. Change in serum $\mathrm{KL}-6$ level from baseline is useful for predicting life-threatening EGFRTKIs induced inter bronchiolar clara cells play a critical role in lung homoeostasis interstitial lung disease. Respir Res. 2011;12:97.

17. Ohnishi $\mathrm{H}$ et al. Circulating KL-6 levels in patients with drug induced pneumonitis. Thorax. 2003;58(10):872-5.

18. Doishita $\mathrm{S}$ et al. Serum KL-6 leve as an indicator of active or inactive interstitial pneumonitis associated with connective tissue diseases. Intern Med. 2011;50(23):2889-92.

19. Jiang $Y$ et al. Sequential changes of serum KL-6 predict the progression of interstitial lung disease. J Thorac Dis. 2018;10(8):4705-14.

20. Fazlyeva RM et al. [Clinical and diagnostic value of the determination of alveomucin in patients with community-acquired pneumonia]. Klin Lab Diagn. 2010;3:51-3. (In Russian).

21. Zheng $D$ et al. A cellular pathway involved in clara cell to alveolar Type II cell differentiation after severe lung injury. PLoS One. 2013;8(8):e71028. 
22. Doubková M et al. Prognostic significance of surfactant protein $A$, surfactant protein $D$, clara cell protein $16, \mathrm{~S} 100$ protein, trefoil factor 3 , and prostatic secretory protein 94 in idiopathic pulmonary fibrosis, sarcoidosis, and chronic pulmonary obstructive disease. Sarcoidosis Vasc Diffuse Lung Dis. 2016;33(3):224-34
23. Petrek $\mathrm{M}$ et al. Clara cell protein (CC16) in serum and bronchoalveolar lavage fluid of subjects exposed to asbestos. Biomarkers. 2002;7(1):5867.

24. Wang SX et al. Roles of serum clara cell protein 16 and surfactant protein- $\mathrm{D}$ in the early diagnosis and progression of silicosis. J Occup Environ Med. 2007;49(8):834-9.

25. Hałatek $T$ et al. Serum clara cell protein as an indicator of pulmonary impairment in occupational exposure at aluminum foundry. Int J Occup Med Environ Health. 2006;19 (4):21123. 